\title{
Superparamagnetic Iron Oxide Nanoparticles (SPIONs) as Cores for Molecularly Imprinted Polymers (MIP) in Trace Analysis
}

\author{
Maria Guć ${ }^{1}$, Grzegorz Schroeder ${ }^{1}$ \\ ${ }^{1}$ Adam Mickiewicz University in Poznań, Faculty of Chemistry, \\ Uniwersytetu Poznańskiego 8, 61-614 Poznań, Poland \\ maria.guc@amu.edu.pl,schroede@amu.edu.pl
}

\begin{abstract}
The following article presents an effective method of core-shell systems preparation, utilizing superparamagnetic iron oxide nanoparticles (SPIONs) as the core. In this research, various molecularly imprinted polymers (MIP) were used as the shell. Obtained system combines magnetic properties of the iron oxide nanoparticles and selective analytical properties of the polymeric coating. Resulting magnetic molecularly imprinted polymers (mag-MIP) were used for initial concentration and trace analysis of organic compounds in environmental samples. SPIONs modified with TEOS (tetraethoxysilane) and MPS (3- (trimethoxysilyl) propyl methacrylate) were used as a magnetic core. EGDMA (ethylene glycol dimethacrylate) and AIBN (2,2'-azobisisobutyronitrile) were used as a crosslinking agent in thermal polymerization. Different classes of compounds were used as polymer matrices: flavonoids, herbicides, pesticides, hormones, for which the appropriate monomers were selected. Mag-MIP was successfully used to determine all tested chemicals in environmental samples. Trace amounts of analytes were adsorbed from their solutions onto the surface of functionalised SPIONs. Subsequently, mag-MIP were attracted by magnets immersed in the solutions and analysed via electrospray ionization mass spectrometry (ESI-MS) and flowing atmospheric pressure afterglow mass spectrometry (FAPA-MS) combined with thermally initiated desorption. Mag-MIP combined with FAPA-MS is a novel analytical method suitable for trace detection from highly heterogeneous solutions. The combination of an analyte pre-concentration with mag-MIP followed by FAPA-MS analysis significantly reduced limit of detection (LOD) for all trace analyses.
\end{abstract}

Keywords: Nanomaterials, Superparamagnetic iron oxide nanoparticles (SPIONs), $\mathrm{Fe}_{3} \mathrm{O}_{4}$, molecularly imprinted polymers (MIP), Magnetic molecularly imprinted polymers (mag-MIP), Sample pre-treatment, Trace analysis, Flowing atmosphericpressure afterglow mass spectrometry (FAPA-MS)

\section{Introduction}

The technology of MIP production is based on the formation of complexes between the analyte/template/guest molecule and the functional monomer/host molecule (Fig. 1). The technique involves polymerization of a functional monomers and a cross-linker around the template. The analyte-monomer complex is then subjected to polymerization initiated by temperature or irradiation (thermal or photoinitiation) during which a three-dimensional polymer structure

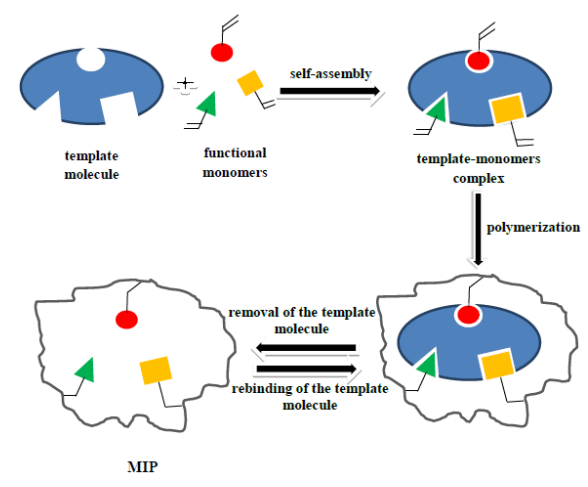

Fig. 1: General scheme of obtaining MIP. is formed. At the subsequent stage, the template is removed from the polymer structure, leaving empty cavities capable of binding molecules similar to the template in size, shape and functional group positions. The use of organic porogens makes the polymer hydrophobic. The polymers obtained are chemically stable, water insoluble (which permits their multiple use in chemical analysis) and their synthesis is relatively simple, reproducible and cheap. However, one of the drawbacks of MIP stems from the need to isolate the dispersed analyte from the solution. It is necessary to use several stages of filtration or stirring, which is costintensive and time-consuming. This can be overcome by the introduction of a magnetic core into the polymer structure, which will allow for magnetic isolation. This solution not only helped to deal with the main problem, but also gave additional benefits. Obtained mag-MIP retain their magnetic properties, and thus can still be easily manipulated by external magnetic fields. Additionally, the core provides defined particle size and shape which results in a higher surface area in 
comparison to MIP. Moreover, the recognition sites are located on the surface of the material, facilitating the analyte binding. Due to their magnetic and surface properties, mag-MIP are more efficient than MIP obtained by traditional synthesis methods. These advantages of mag-MIP render them highly attractive for a wide variety of applications in separation techniques. Mag-MIP was used for the analysis of pharmaceutical compounds, flavonoids, herbicides, pesticides, hormones, estrogens in environmental samples. The goal of our research was to perform trace analysis from samples forming a complex matrix, so it was important to shorten the analytical stages at which the analyte could be lost [1-5]. Classical removal of the analyte molecule from polymeric structures and ESI-MS analysis has been replaced by direct FAPA-MS analysis. Flowing atmospheric pressure afterglow mass spectrometry (FAPA-MS) is a technique of analytical chemistry for sensitive detection of low molecular weight small organic compounds. The method of analysis by mass spectrometry with the flowing atmospheric pressure afterglow ion source in the ambient atmosphere has been proven to be a promising tool for direct and rapid determination of many compounds. The construction of a helium plasma torch and its operating parameters have a direct impact on the quality of the spectra obtained with the mass spectrometer.

a)

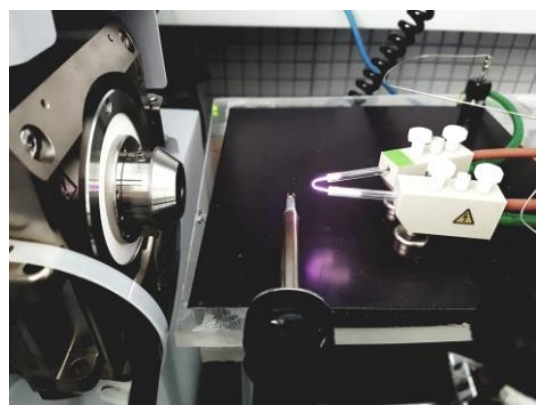

b)

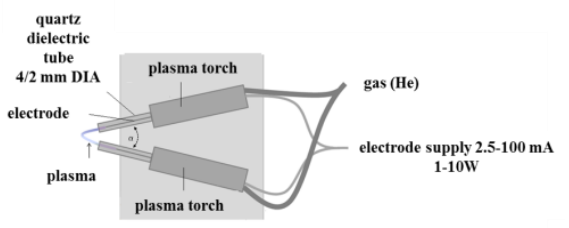

c)

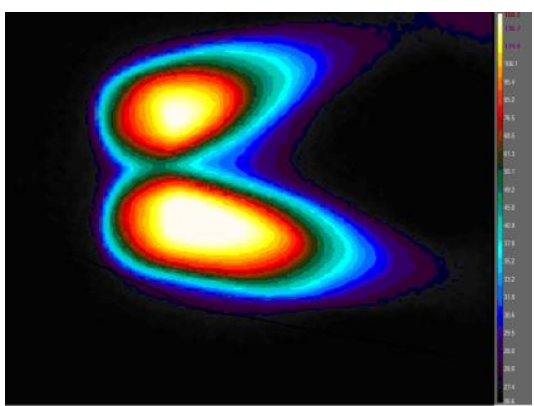

Fig. 2: a) The system used to carry out the experiment (FAPA-MS); b) FAPA ambient plasma source; c) Plasma temperature distribution in the FAPA burner.

The helium plasma is obtained between the 2 electrodes inside a quartz tube. The first electrode is inside quartz tube and the second electrode is outside at the end of the quartz tube. The analytes were thermally desorbed and ionized with FAPA and identified in a classic way with the mass spectrometer analyzer. Thermal desorption of analytes is a specific method of introducing a sample into the plasma stream. The order of release of the compounds depends on their volatility. The method offers fast and reliable structural information, with no need of pre-separation, and can be an alternative to the following techniques: EI-MS, GC-MS, ESI-MS; for fast analysis of organic compounds [6-7].

This paper presents new analytical procedures for determination of organic compounds: flavonoids, herbicides, pesticides, hormones in real samples with the use of mag-MIP for pre-concentration and flowing atmospheric-pressure afterglow mass spectrometry (FAPA-MS) with thermal desorption of analytes. The combination of analyte preconcentration technique with mag-MIP and FAPA-MS analysis is a novel method with decreased LOD for several trace analytes.

\section{Experimental}

\subsection{Materials and chemicals}

All reagents used were commercial products. $\mathrm{FeCl}_{2} \cdot 4 \mathrm{H}_{2} \mathrm{O}, \mathrm{FeCl}_{3} \cdot 6 \mathrm{H}_{2} \mathrm{O}$, tetraethoxysilane (TEOS), hydrochloric acid $(\mathrm{HCl})$, citric acid, sodium hydroxide $(\mathrm{NaOH})$, ammonia solution $(\mathrm{NH} 4 \mathrm{OH})$, acrylamide (AA), methacrylic acid (MA), ethylene glycol dimethacrylate (EGDMA), 2,2'-azobisisobutyronitrile solution $0.2 \mathrm{M}$ in toluene (AIBN), Aluminum Oxide Activated, basic, Brockman I, 3-metacriloxipropiltrimetoxissilano (MPS), atrazine, pendimethalin, quercetin (Q), estrone (E1), $\beta$-estradiol (E2) and all solvents: toluene, chloroform, N,N-Dimethylformamide (DMF), ethanol, acetic acid of the purity grade p.a., were obtained from Sigma-Aldrich (St. Louis, MO, USA). 


\subsection{Instruments}

The material was tested by Fourier Transform Infrared (FT-IR) spectroscopy and thermogravimetric analysis (TGA). The infrared spectra were taken with IFS $66 \mathrm{v} / \mathrm{s}$ FTIR spectrophotometer from Bruker, equipped with an MCT detector. The spectra were recorded in the $400-4000 \mathrm{~cm}^{-1}$ range for $\mathrm{KBr}$ pellets. Functionalization of the obtained materials was confirmed via TGA. The measurements were performed on Setsys 1200 thermogravimetric analyzer (Setaram) with a heating rate of $10^{\circ} \mathrm{C} \mathrm{min}^{-1}$ from room temperature to $1000^{\circ} \mathrm{C}$ in helium atmosphere. Scanning electron microscopy (SEM) images were acquired with QUANTA 250 FEG, FEI.

FAPA-MS spectra were recorded using amaZon SL ion trap (Bruker, Bremen, Germany) equipped with FAPA ambient plasma source. The apparatus was operated using the so-called "enhanced resolution mode" (mass range: 50$2,200 \mathrm{~m} / z$, scanning rate: $8,100 \mathrm{~m} / z$ per second). The capillary voltage was set at $-4.5 \mathrm{kV}$ and the endplate offset at $-500 \mathrm{~V}$. Helium was used as the cone gas and desolvating gas (nitrogen) at flow rates of $50 \mathrm{~L} \mathrm{~h}^{-1}$ and $800 \mathrm{~L} \mathrm{~h}^{-1}$, respectively. The mass spectrometer was operated in the positive ionization mode. In $\mathrm{MS}^{n}$ experiments, the width of the selection window was set at $2 \mathrm{Da}$ and the amplification of the excitation was set according to the experiment (from 0.2 to $1.5 \mathrm{~V}$ ) [8-10].

\subsection{Synthesis of magnetic molecularly imprinted polymers \\ - Synthesis of Fe304 as magnetic core}

The magnetite nanoparticles $\left(\mathrm{Fe}_{3} \mathrm{O}_{4}\right)$ were synthesized in a two-step procedure. Magnetite $\left(\mathrm{Fe}_{3} \mathrm{O}_{4}\right)$ nanoparticles were synthesized via precipitation method. $\mathrm{FeCl}_{2} \cdot 4 \mathrm{H}_{2} \mathrm{O}$ and $\mathrm{FeCl}_{3} \cdot 6 \mathrm{H}_{2} \mathrm{O}$ were used as ferric and ferrous ions precursors. $\mathrm{FeCl}_{2} \cdot 4 \mathrm{H}_{2} \mathrm{O}(2 \mathrm{~g})$ and $\mathrm{FeCl}_{3} \cdot 6 \mathrm{H}_{2} \mathrm{O}(5.2 \mathrm{~g})$ were dissolved in $25 \mathrm{ml}$ of deoxygenated water, and then $0.85 \mathrm{ml}$ of concentrated $\mathrm{HCl}$ were added. The resulting solution was added dropwise into $250 \mathrm{ml}$ of $1.5 \mathrm{M} \mathrm{NaOH}$ solution upon vigorous stirring in $\mathrm{N}_{2}$ atmosphere at $80^{\circ} \mathrm{C}$. The synthesized SPIONs were separated from the solution with a magnet and washed with $200 \mathrm{ml}$ of deionized water three times.

\section{- Modification of the magnetic core}

The surface-functionalized magnetite was produced via hydrolysis and condensation of the TEOS organosilane agents. Firstly, $10 \mathrm{ml}$ of an aqueous solution of citric acid $\left(0.5 \mathrm{~g} \mathrm{ml}^{-1}\right)$ was added to the rigorously stirred suspension of washed nanoparticles. Then, the $\mathrm{pH}$ was set to 5.2 with concentrated ammonia solution and heated to $80^{\circ} \mathrm{C}$. After $90 \mathrm{mins}$, the $\mathrm{pH}$ value of the solution was elevated to 10 , using $\mathrm{NaOH}$ solution. Subsequently, the suspension was centrifuged for 5 mins at $4000 \mathrm{rpm}$ to remove any agglomerated nanoparticles. Next, silica coat was fabricated on the surface of $\mathrm{Fe}_{3} \mathrm{O}_{4} \mathrm{MNPs}$ through a sol-gel method. $100 \mathrm{ml}$ of ethanol containing $4 \mathrm{ml}$ of TEOS were added in the above prepared stable aqueous suspension of $\mathrm{Fe}_{3} \mathrm{O}_{4} \mathrm{MNPs}$, followed by stirring at ambient temperature for $6 \mathrm{~h}$. After rinsing with ethanol and water several times, the obtained $\mathrm{Fe}_{3} \mathrm{O}_{4} @ \mathrm{SiO}_{2} \mathrm{MNPs}$ were dried at $60^{\circ} \mathrm{C}$. In the next step, $\mathrm{Fe}_{3} \mathrm{O}_{4} @ \mathrm{SiO}_{2}$ was modified with MPS. For this purpose $250 \mathrm{mg}$ of obtained material were dispersed in $50 \mathrm{~mL}$ of anhydrous toluene containing $5 \mathrm{~mL}$ of MPS and the mixture was allowed to react at $60^{\circ} \mathrm{C}$ for $24 \mathrm{~h}$ under dry nitrogen. The mixture was filtered through a membrane, washed with toluene and dried in vacuum. Finally, the silanized and modified material $\mathrm{Fe}_{3} \mathrm{O}_{4} @ \mathrm{SiO}_{2}-\mathrm{MPS}$ was obtained.

\section{- Synthesis of molecularly imprinted polymers with magnetic core}

The next step involved the preparation of a selective mag-MIP towards chosen analyte. For instance, mag-MIP selective for quercetin was prepared by polymerization of $0.2 \mathrm{mmol}$ template-Q and $0.8 \mathrm{mmol}$ functional monomer-AA in $30 \mathrm{~mL}$ of a porogenic solvent - ethanol, a portion of $0.4 \mathrm{mmol}$ of the template molecule-atrazine and 1.6 mmol functional monomer-MAA were dissolved in $25 \mathrm{ml}$ of methanol, while $0.6 \mathrm{mmol}$ of the template molecule-pendimethalin and 2.4 mmol the same functional monomer were dissolved in $10 \mathrm{ml}$ chloroform. The mixture was placed in ultrasound bath at $25^{\circ} \mathrm{C}$ for $1 \mathrm{~h}$. Then, $200 \mathrm{mg}$ of $\mathrm{Fe}_{3} \mathrm{O}_{4} @ \mathrm{SiO}_{2}$-MPS were added into the mixture, which was sonicated for another $1 \mathrm{~h}$. Subsequently, $4.0 \mathrm{mmol}$ of the cross-linking agent-EGDMA and $1 \mathrm{ml}$ of the initiator-AIBN were added into the system and the mixture was sonicated at $80^{\circ} \mathrm{C}$ for $24 \mathrm{~h}$. Mag-MIP selective for hormones (estrone, $\beta$-estradiol) were prepared in the same manner, by polymerization of $1 \mathrm{mmol}$ template-E1 or E2 and 4 mmol functional monomer-MA in $30 \mathrm{~mL}$ of a same porogenic solvent-ethanol. The mixture was placed in ultrasound bath at $25^{\circ} \mathrm{C}$ for $1 \mathrm{~h}$. Then $200 \mathrm{mg}$ of $\mathrm{Fe}_{3} \mathrm{O}_{4} @ \mathrm{SiO}_{2^{-}}$ MPS were added into the system and the mixture was sonicated for another $1 \mathrm{~h}$. Next, $20 \mathrm{mmol}$ of the cross-linking agentEGDMA and $1 \mathrm{ml}$ of the initiator-AIBN were added into the system and the mixture was sonicated at $80^{\circ} \mathrm{C}$ for $24 \mathrm{~h}$. All obtained materials were dried under reduced pressure and grounded. Afterwards, the templates were removed by Soxhlet 
extraction using a mixture of ethanol: acetic acid (9:1, v:v) as eluent for few days. The eluent was exchanged every day. Finally selective mag-MIP were dried and grounded again.

The levels of template elution were controlled with FAPA-MS analysis of the dry mag-MIP. It was determined that after the extraction, materials containing about $1 \%$ and $5 \%$ of the template in the mag-MIP were obtained. Further extraction or solvent substitution did not change the template elution level.

As a control experiment, magnetic non-imprinted polymers (mag-NIP) were also prepared and treated in the identical manner, excluding utilization of template structures during polymerisation process.

\subsection{Trace analysis of organic compounds with the use magnetic molecularly imprinted polymers}

As already mentioned in the introduction, there are 2 ways of analytical use of magnetic molecularly imprinted polymers.

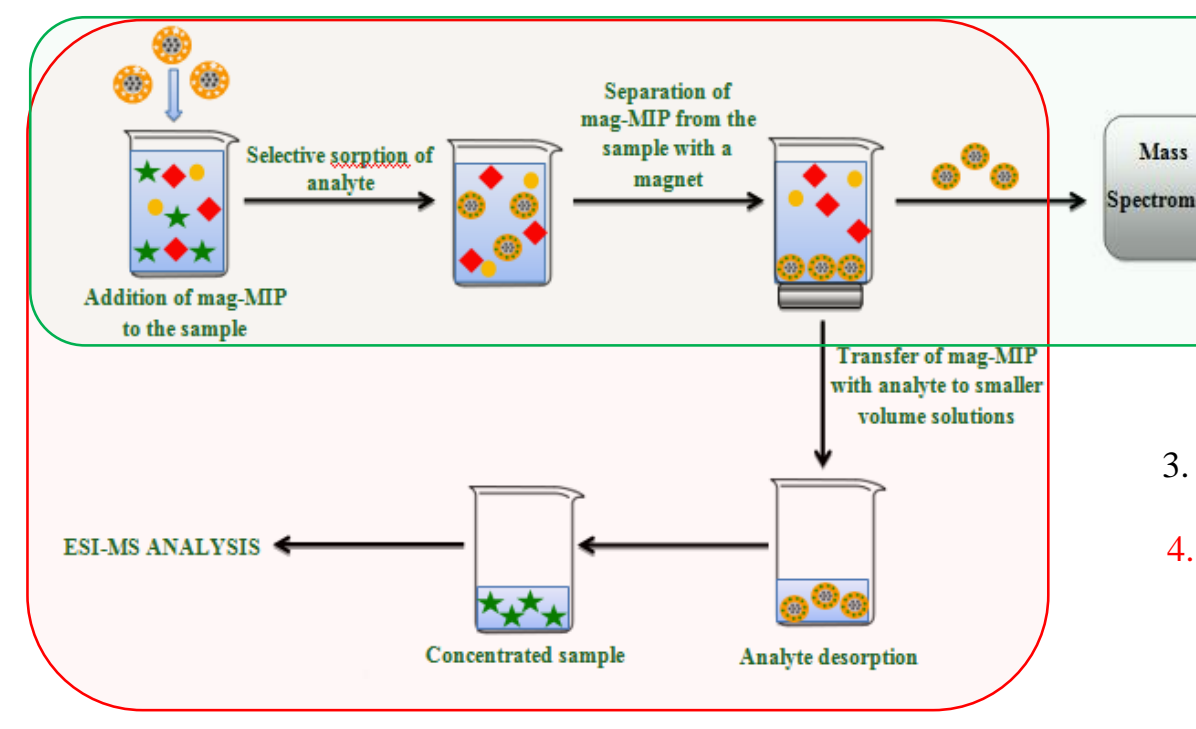

4 STEPS in $1 \longrightarrow$ FAPA-MS analysis

\section{Results and Discussion}

\subsection{Characterization of mag-MIP}

\section{- FT-IR analysis}

FT-IR spectra confirmed the binding of the template in mag-MIP polymer structures. Ascending bonds were only found in the mag-NIP systems. In addition, they allow to control the process of washing the template from the polymer.

- TG analysis

TG analysis shows the weight loss of the material due to decomposition of the built-in template caused by the temperature increase. This analysis allows to confirm the presence of a given template, determine its residual content in the polymer structure and provide optimal analysis temperature for the FAPA-MS method. This temperature usually coincides with the melting point of the analyte. In all cases, the polymeric material is stable up to a temperature ca. $300^{\circ} \mathrm{C}$.

\section{- SEM images}

SEM images reveal the morphology of the obtained materials and allow to determine their shape and size. All images showed that the obtained magnetic products have spherical morphology and a diameter of less than $100 \mathrm{~nm}$. The following is a set of analyzes made for materials used for estrogens determination. 

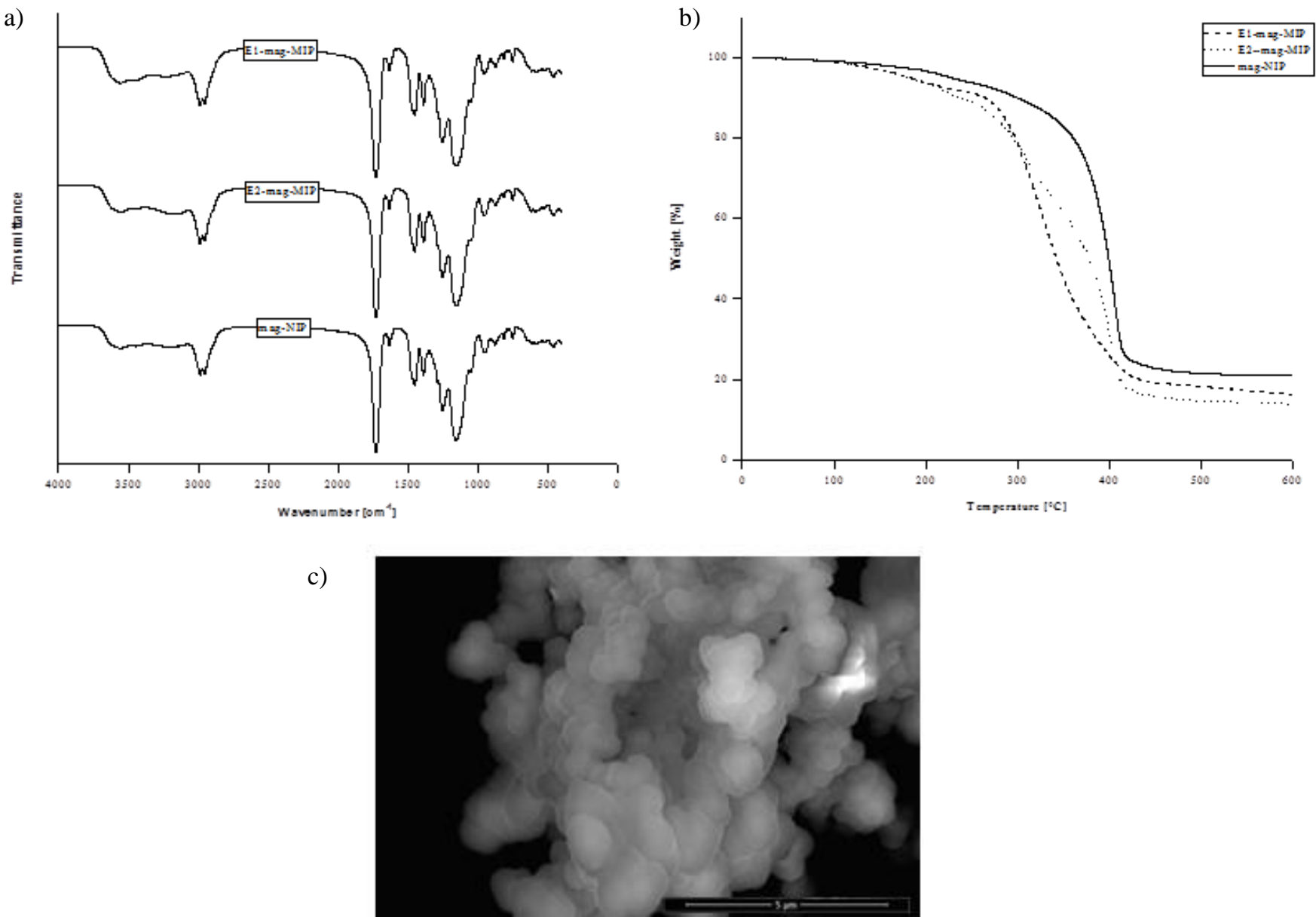

Fig. 3: a) The FT-IR spectra of: E1-mag-MIP, E2-mag-MIP, mag-NIP; b) Thermogravimetric analysis of: E1-mag-MIP, E2-mag-MIP, mag-NIP; c) SEM imagine of E2-mag-MIP after washing template.

\subsection{FAPA-MS analyses}

\section{- LOD determination procedure}

All experiments were repeated three times. The relative standard deviation (RSD) obtained for all data did not exceed $10 \%$. In order to determine the background signals, the spectra of particular matrices (NIPs) without the analytes were recorded. The limit of detection (LOD) is the concentration of a substance below which its identity cannot be distinguished from analytical artefacts. The limit of detection (LOD) was calculated according to the definition: $\mathrm{LOD}=$ mean blank value $+3 \times$ standard deviation. The $\mathrm{m} / \mathrm{z}$ signal of the analyte was three times higher than that of the noise level. In the experiments in which the LOD of composite matrices were determined, $100 \mathrm{mg}$ of the mixture of mag$\mathrm{MIP} / \mathrm{mag}$-NIP with different concentrations of the analytes were introduced into the crucible and also heated to ca. $200^{\circ} \mathrm{C}$.

\section{- Linear function analysis}

In the context of an analysis system, the linearity means that we assume a linear relationship between the concentration of analyte $(x)$ and intensity of $\mathrm{m} / \mathrm{z}$ signals $(y)$, which can be written mathematically as $y=f(x)$. It is a common practice to check the linearity of a calibration curve by inspection of the correlation coefficient $r$. A correlation coefficient close to unity $(r=1)$ is considered sufficient evidence to conclude that a perfect linear calibration has been achieved. Using different weights of the solid matrices with the analyzed compounds, it was found that the linearity of the analyte determinations was satisfied when the correlation coefficient of the function $y=f(x)$ was greater than 0.980 . 


\section{- Determination of small organic compounds in water/sludge using mag-MIP and FAPA-MS method}

A portion of $10 \mathrm{mg}$ sample-specific mag-MIP was added to the water/sludge with analyte. A solution with the addition of mag-MIP was mixed for 5 minutes. Then, magnetic core containing mag-MIP was isolated using a neodymium magnet. Subsequently, polymers were dried in open air. Portions of $10 \mathrm{mg}$ of polymers with absorbed analyte were placed on the apparatus heating table, heated and then the FAPA-MS spectra were recorded. The use of this technique allows for the analysis of trace amounts of low molecular weight organic compounds from very complex materials such as wastewater. The following spectra confirm a lack of interference from other compounds in the sample.
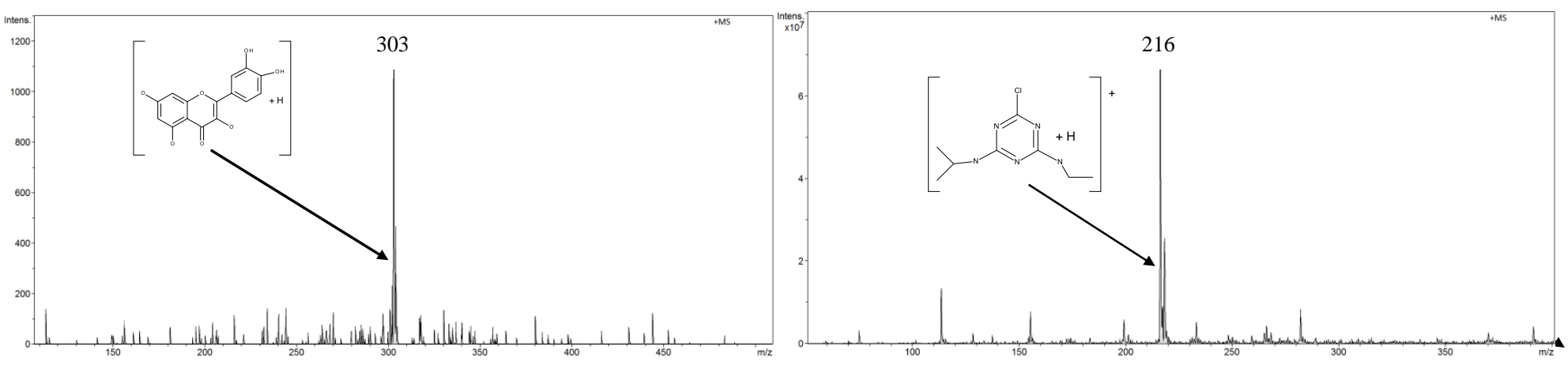

Fig. 4: FAPA positive ions spectrum of quercetin released directly from quercetin-mag-MIP and atrazine from atrazine-mag-MIP.

Table 1 presents the linearity range and the LOD for the analyzed compounds.

Table 1: Analytical parameters.

\begin{tabular}{|c|c|c|}
\hline Analyte & $\begin{array}{c}\text { Linear range } \\
{[\mathbf{n M}]}\end{array}$ & $\begin{array}{c}\text { LOD } \\
{[\mathbf{n M}]}\end{array}$ \\
\hline Atrazine & $3-200$ & 1 \\
\hline Pendimethalin & $10-200$ & 1 \\
\hline Quercetin & $30-200$ & 10 \\
\hline Estrone & $1-200$ & 0.5 \\
\hline$\beta$-estradiol & $1-200$ & 0.5 \\
\hline
\end{tabular}

Described method was applied for determination of quercetin using FAPA-MS analysis, in water solutions of capers and onion. Performed studies indicate detection limits at 0.00055 and $0.000049 \mathrm{M}$, respectively. Recalculation among the dry mass of capers and onion gave a quercetin content of 158 and $17 \mathrm{mg} / 100 \mathrm{~g}$, respectively. Furthermore, the results of the conducted research have shown that the technique applied allows for determination of estrogens in water at concentration $10^{-9} \mathrm{M}\left(0.271 \mu \mathrm{g} \mathrm{L}^{-1}\right)[9-11]$.

\section{Conclusion}

In conclusion, we explored synthesis of different molecularly imprinted polymers coated $\mathrm{Fe}_{3} \mathrm{O}_{4}$ magnetic nanoparticles that exhibit a much higher specific recognition and saturation magnetization. A new method for determination of organic compounds based on the use of mag-MIP dedicated for this analyte and their FAPA-MS analysis was proposed. The range of this method application was established. The use of the FAPA-MS method, a modern tool enabling one-step, direct testing of organic substances contained in the solid phase, allowed not only for their detection, but also to reduce the operational stages in the analytical way. In addition, the use of mag-MIP provided a method for efficient isolation of the analyte from the sample and its stable storage. This is extremely useful for unstable compounds such as 
estrogens. The combination of molecular imprinting technique and FAPA-MS technique has created a rapid analytical method for the determination of bioactive substances with molecular weight up to $500 \mathrm{Da}$ in the solid phase.

\section{Acknowledgements}

This work was supported by National Grant no. 2016/21/B/ST4/02082, provided by the National Science Centre.

\section{References}

[1] M. Guć and G. Schroeder, "The Molecularly Imprinted Polymers. Influence of Monomers on the Properties of Polymers -A Review," World Journal of Research and Review, vol. 5, no. 6, pp. 36-47, 2017.

[2] R. J. Uzuriaga-Sanchez, S. Khan, A. Wong, G. Picasso, M. I. Pividori and M. del Pilar Taboada Sotomayor, "Magnetically separable polymer (Mag-MIP) for selective analysis of biotin in food samples," Food Chemistry, no. 190, pp. 460-467, 2016.

[3] X. Wang, L. Wang, X. He, Y. Zhang and L. Chen, "A molecularly imprinted polymer-coated nanocomposite of magnetic nanoparticles for estrone recognition," Talanta, vol. 78, no. 2, pp. 327-332, 2009.

[4] H. Lu and S. Xu, "Mesoporous structured estrone imprinted Fe3O4@SiO2@mSiO2 for highly sensitive and selective detection of estrogens from water samples by HPLC," Talanta, vol. 144, no. 1, pp. 303-311, 2015.

[5] Q. Han, X. Shen, W. Zhu, C. Zhu, X. Zhou and H. Jiang, "Magnetic sensing film based on Fe3O4@Au-GSH molecularly imprinted polymers for the electrochemical detection of estradiol," Biosensors and Bioelectronics, vol. 79, no. 1, pp. 180-186, 2016.

[6] A. Albert, J. T. Shelley and C. Engelhard, "Plasma-based ambient desorption/ionization mass spectrometry: state-ofthe-art in qualitative and quantitative analysis," Analytical and Bioanalytical Chemistry, vol. 406, no. 25, pp. 61116127, 2014.

[7] M. Brüggemann, E. Karu and T.Hoffmann, "Critical assessment of ionization patterns and applications of ambient desorption/ionization mass spectrometry using FAPA-MS," Journal of Mass Spectrometry, vol. 51, no. 2, pp. 141$149,2016$.

[8] M. Cegłowski, M. Smoluch, E. Reszke, J. Silberring and G. Schroeder, "Molecularly imprinted polymers as selective adsorbents for ambient plasma mass spectrometry," Analytical and Bioanalytical Chemistry, vol. 409, no. 13, pp. 3393-33405, 2017.

[9] M. Guć, E. Reszke, M. Cegłowski and G. Schroeder, "Construction of Plasma Ion Sources to be Applied in Analysis of Small Organic Compounds Using Mass Spectrometry," Plasma Chemistry and Plasma Processing, 2019.

[10] M. Guć, E. Reszke, M. Cegłowski and G. Schroeder, "The Application of the Microwave Plasma Ionization Source in Ambient Mass Spectrometry," Plasma Chemistry and Plasma Processing, vol. 39, no. 4, pp. 1001-1017, 2019.

[11] M. Guć and G. Schroeder, "Application of Molecularly Imprinted Polymers (MIP) and Magnetic Molecularly Imprinted Polymers (mag-MIP) to Selective Analysis of Quercetin in Flowing Atmospheric-Pressure Afterglow Mass Spectrometry (FAPA-MS) and in Electrospray Ionization Mass Spectrometry (ESI-MS)," Molecules, vol. 24, no. 13, pp. 2364-2379, 2019. 